\title{
Non-equilibrium ionization modeling of the Local Bubble
}

\section{Tracing Civ, Nv, and Ovı ions}

\author{
M. A. de Avillez ${ }^{1}$ and D. Breitschwerdt ${ }^{2}$ \\ 1 Department of Mathematics, University of Évora, R. Romao Ramalho 59, 7000 Évora, Portugal \\ e-mail: mavillez@galaxy.lca.uevora.pt \\ 2 Zentrum für Astronomie und Astrophysik, Technische Universität Berlin, Hardenbergstrasse 36, 10623 Berlin, Germany \\ e-mail: breitschwerdt@astro.physik.tu-berlin.de
}

Received 2 May 2011 / Accepted 23 January 2012

ABSTRACT

\begin{abstract}
Aims. We present the first high-resolution non-equilibrium ionization simulation of the joint evolution of the Local Bubble (LB) and Loop I superbubbles in the turbulent supernova-driven interstellar medium (ISM). The time variation and spatial distribution of the Li-like ions Civ, Nv, and Ovi inside the LB are studied in detail.

Methods. This work uses the parallel adaptive mesh refinement code EAF-PAMR coupled to the newly developed atomic and molecular plasma emission module $\mathrm{E}(\mathrm{A}+\mathrm{M}) \mathrm{PEC}$, featuring the time-dependent calculation of the ionization structure of $\mathrm{H}$ through Fe, using the latest revision of solar abundances. The finest AMR resolution is $1 \mathrm{pc}$ within a grid that covers a representative patch of the Galactic disk (with an area of $1 \mathrm{kpc}^{2}$ in the midplane) and halo (extending up to $10 \mathrm{kpc}$ above and below the midplane).

Results. The evolution age of the LB is derived by the match between the simulated and observed absorption features of the Li-like ions Civ, Nv, and Ovi. The modeled LB current evolution time is bracketed between 0.5 and $0.8 \mathrm{Myr}$ since the last supernova reheated the cavity in order to have $N(\mathrm{OvI})<8 \times 10^{12} \mathrm{~cm}^{-2}, \log [N(\mathrm{CIV}) / N(\mathrm{OvI})]<-0.9$ and $\log [N(\mathrm{Nv}) / N(\mathrm{OvI})]<-1$ inside the simulated LB cavity, as found in CoPERNICUs, IUE, GHRS-IST and FUSE observations.
\end{abstract}

Key words. hydrodynamics - ISM: structure - ISM: kinematics and dynamics - ISM: bubbles

\section{Introduction}

The ionization structure of the Local Bubble (LB), like that of any superbubble, should in principle reflect its evolutionary state for given boundary (and initial) conditions, if we assume a multi-supernova/stellar wind origin (e.g, Innes \& Hartquist 1984; Cox \& Anderson 1982; Smith \& Cox 2001). While the ambient medium at the time of the first supernova (SN) explosion is unknown, the subsequent time-dependent energy input rate is - quite surprisingly - much more tightly constrained, although no early-type stars (or relics thereof) have been found within the current LB volume. The kinematic analysis of nearby stars (Maíz-Apellániz 2001), moving groups (Berghöfer \& Breitschwerdt 2002; BB02), and the whole volume of $400 \mathrm{pc}$ in diameter centered on the Sun from Hipparcos data (Fuchs et al. 2006) consistently point to 14-19 stars having exploded during the past $15 \mathrm{Myr}$ on their way through the region now occupied by the LB.

Collisional ionization equilibrium (CIE) simulations of the LB powered by the missing stars of the subgroup B1 of Pleiades (BB02) within a turbulent interstellar medium (Breitschwerdt \& de Avillez 2006, BA06; de Avillez \& Breitschwerdt 2009, $\mathrm{AB} 09)$ reproduced the clumpy distribution of OvI, as well as its column density and dispersion inside the LB cavity observed by Copernicus (Jenkins 1978; Shelton \& Cox 1994) and FUSE (Oegerle et al. 2005; Savage \& Lehner 2006), and the lack of $\mathrm{CIV}$ and $\mathrm{Nv}$ ions in the bubble, which is consistent with the observations with Copernicus (York 1977), IUE (Bruhweiler et al. 1980), and GHRS-HST (Bertin et al. 1995; Huang et al. 1995). These observations point to column densities $N($ CIV $)<7 \times 10^{11}, N(\mathrm{Nv})<3 \times 10^{12} \mathrm{~cm}^{-2}$, $\log [N(\mathrm{CIV}) / N(\mathrm{OvI})] \lesssim-0.9$ and $\log [N(\mathrm{Nv}) / N(\mathrm{OvI})] \lesssim-1.0$. In that CIE model, the match between the simulated and observed column densities indicated that the last SN event occurred 0.5 Myr ago.

However, significant deviations from global CIE conditions are likely to occur (e.g., Kafatos 1973) as suggested by observations of the LB in the EUV (Jelinsky et al. 1995; Hurwitz et al. 2005) and X-ray (Sanders et al. 2001; McCammon et al. 2002; Freyberg \& Breitschwerdt 2003; Henley et al. 2007) bands and by non-equilibrium ionization (NEI) one-dimensional simulations of the LB (e.g., Cox \& Anderson 1982; Smith \& Cox 2001).

The present work explores the degree to which nonequilibrium ionization (NEI) affects the conclusions of otherwise similar CIE modeling. We extend our previous CIE work (BA06 and AB09) to (i) model the LB evolution hydrodynamically and trace its ionization structure (and that of the surrounding ISM) in a time-dependent fashion; (ii) study the spatial distribution of the Li-like ions Civ, Nv, and Ovi and compare them with observations; and (iii) constrain the LB age by matching simulated and observed line-of-sight distributions of these ions. This letter is organized as follows: Sect. 2 summarizes the new model setup and simulation. In Sect. 3, the distribution of Lilike ions and their column density ratios within the LB are discussed and compared to observations. The discussion of the results (Sect. 4) closes the paper.

We simulate the effects of the explosions of the stars of a moving subgroup, the living members of which now belong to the UCL and LCC associations, as their trajectories have crossed the LB volume towards their present position. 


\section{Model and numerical setup}

We simulate the simultaneous evolution of the Local and Loop I superbubbles. These are the result of the successive explosions of massive stars from, respectively, a moving subgroup consisting of 17 stars with masses $\in[8.2,21.5] M_{\odot}$, whose living members now belong to the UCL and LCC associations, as their trajectories have crossed the LB volume towards their present position (Fuchs et al. 2006), and Sco Cen consisting of 39 stars with masses $\in[14,31] M_{\odot}$ (Egger 1998). Their time of explosion is calculated by the Fuchs et al. (2006) formula. The simulation setup is similar to that discussed in BA06 and AB09, but with three major differences from these previous CIE models: Firstly, the Time-dependent evolution of the ionization structure of $\mathrm{H}, \mathrm{He}, \mathrm{C}, \mathrm{N}, \mathrm{O}, \mathrm{Ne}, \mathrm{Mg}, \mathrm{Si}, \mathrm{S}$, and Fe ions with Asplund et al. (2009) abundances derived using the Eborae Atomic+Molecular Plasma Emission Code (E(A+M)PEC ${ }^{1}$; Avillez et al. 2011). The physical processes included in $\mathrm{E}(\mathrm{A}+\mathrm{M}) \mathrm{PEC}$ are electron impact ionization, inner-shell excitation auto-ionization, radiative and dielectronic recombination, charge-exchange reactions (recombination with Hi and Her and ionization with Hir and HeIr ), continuum (bremsstrahlung, free-bound, two-photon) and line (permitted, semi-forbidden and forbidden) emission in the range $1 \AA-610 \mu$, and molecular lines. The E(A+M)PEC code also includes inner shell photoionization, ionization of Hı by Lyman continuum photons produced by the recombination of helium, and molecular chemistry involving $\mathrm{H}, \mathrm{C}$, and $\mathrm{O}$. The cooling function (calculated at each cell of the grid at all time steps) includes these processes, while the spectra calculations include line and continuum emissions. The second difference is that local self-gravity and heat-conduction (Balbus \& Mckee 1982; Dalton $\&$ Balbus 1993) are calculated at every time step; Finally, the coarse grid resolution is $8 \mathrm{pc}$, while the finest AMR resolution is $1 \mathrm{pc}-$ the highest so far used for LB evolution calculations. Periodic and outflow boundary conditions are set along the vertical faces and both the top and bottom $(z= \pm 10 \mathrm{kpc})$ of the grid, respectively.

\section{Results}

To model the Local Bubble, which is assumed to be embedded in a realistic background ISM, the simulations were run for a considerable time in order to establish the full Galactic fountain and a global dynamical equilibrium with the density, pressure, and temperature distributions conditions similar to those described in de Avillez \& Breitschwerdt 2004). At an ISM evolution time of $250 \mathrm{Myr}$, a tracker searches for a molecular region of sufficient mass to form the ScoCen stars (adopting a 5\% star formation efficiency). The UCL and LCC clusters paths towards ScoCen are then set using the kinematic parameters derived by Fuchs et al. (2006) with the last SN event in the LB cavity occurring 13.1 Myr after the first explosion (that is, after an ISM evolution time of 263.1 Myr). The Local and Loop I bubbles evolve in time interacting for most of the LB lifetime, and eventually the two bubbles merge with Loop I material expanding into the LB cavity. Here, we discuss the evolution of the LB in the first Myr after the last SN explosion.

\footnotetext{
1 The code is written in OpenCL and runs in AMD and NVIDIA Graphics Processor Units (GPUs). A description of the software and ionization fractions, cooling and emission spectra tables can be found at http://www.lca.uevora.pt/research.html See also references therein.
}

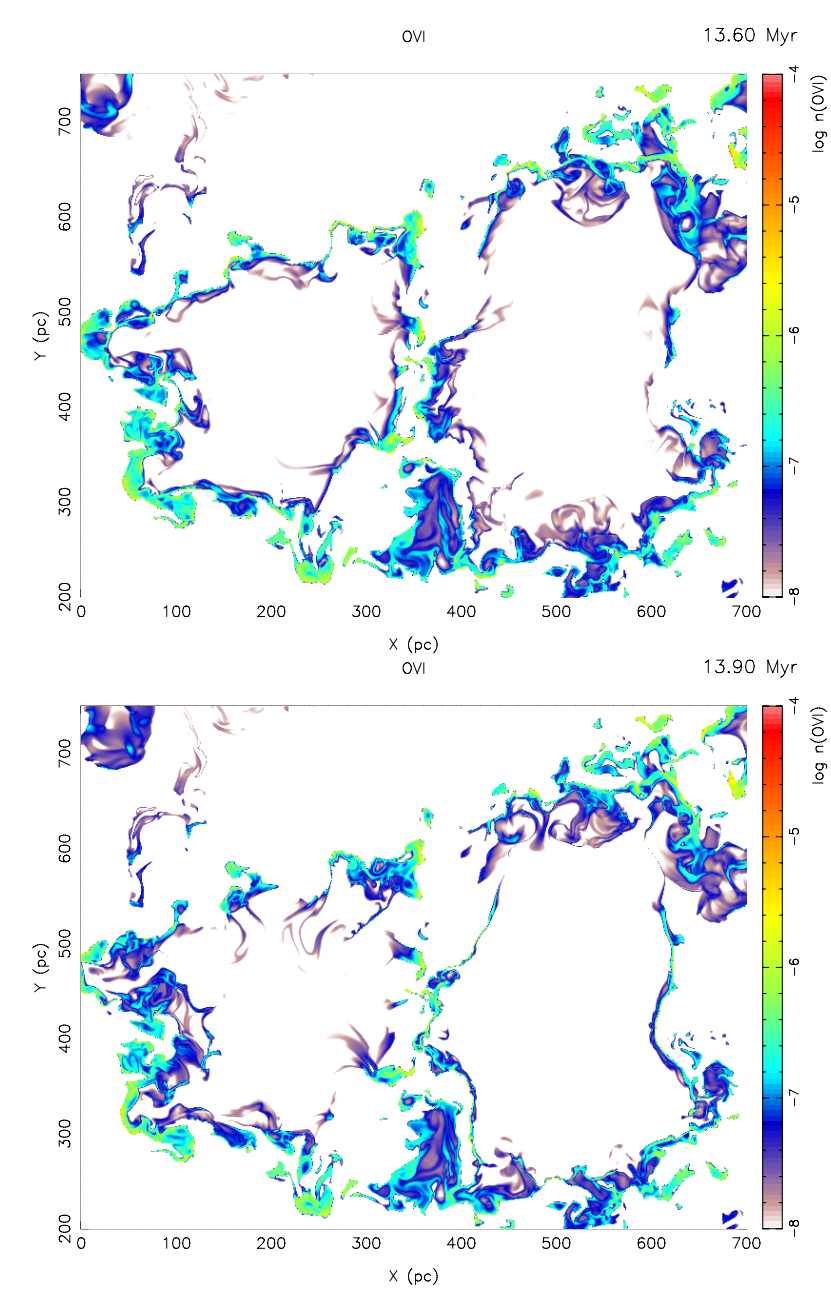

Fig. 1. Ovi density distributions (cuts through the Galactic midplane) in the LB centered at $(x=200, y=450) \mathrm{pc}$ and Loop I (the adjacent bubble on the right) at 0.5 (top) and 0.8 (bottom) Myr after the last SN in the LB, which occurred at evolution time 13.1 Myr. Both bubbles are surrounded with a thin fragmented Ovi bearing shell.

\subsection{Ovı distribution in the $L B$}

Figure 1 displays time snapshots of the Ovi density distribution within the LB at an evolution time (since the first $\mathrm{SN}$ in the LB) of $t_{\mathrm{evol}}=13.6$, and 13.9 Myr, that is $\Delta \tau \in\{0.5,0.8\} \mathrm{Myr}$, respectively, after the last SN event within the LB cavity. The OvI has a clumpy distribution resulting from local cooling and turbulent motions, driven by shear flows inside the LB cavity, whose highest values occur towards the boundary.

The spatial and temporal variations in Ovi are explored by calculating the Ovi column density $(N(\mathrm{OvI}))$ through LOS taken from the Sun at $(x=200, y=450) \mathrm{pc}, 100$ pc from the interaction region between the Local and Loop I bubbles. The column densities were calculated with a 5 pc step length out to a maximum of $200 \mathrm{pc}$, spaced at 1 degree intervals to examine the full $200 \mathrm{pc}$ radius sphere about the assumed Solar position. Figure 2 displays the maximum $N(\mathrm{OvI})$ values over all the lines of sight at times 0.5 Myr through to 1, 1.5 and 2.0 Myr after the last SN explosion in the cavity. In addition, the figure also shows FUSE data towards white dwarfs (Savage \& Lehner 2006, SL2006; Welsh \& Lallement 2008, WL2008; Barstow et al. 2010, BB2010).

The maximum $N(\mathrm{OvI})$ increases with both path length and time, being lower than $10^{13} \mathrm{~cm}^{-2}$ inside the LB up to a radius of 


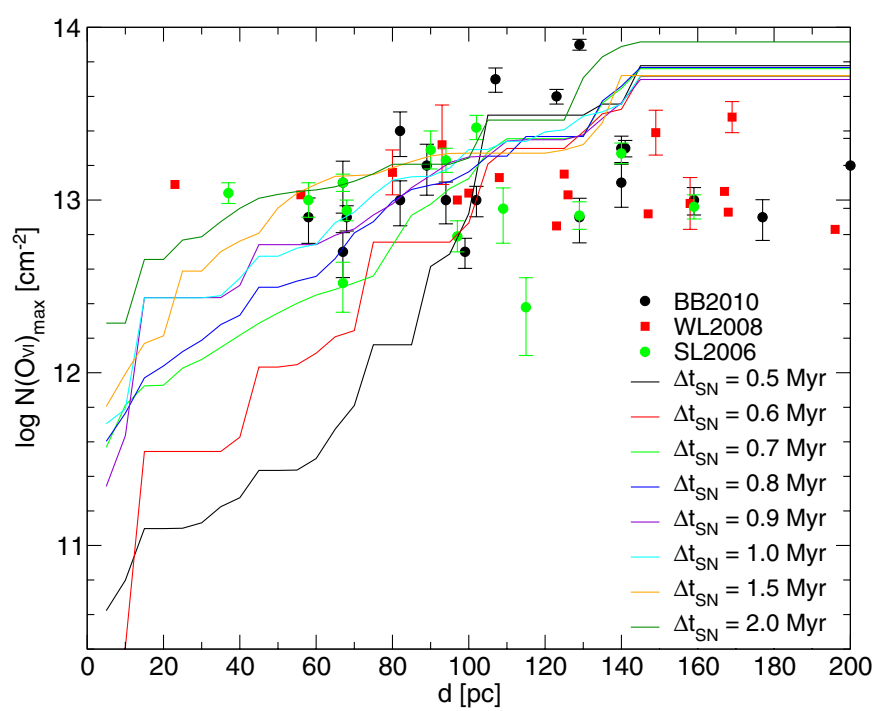

Fig. 2. Maximum (top) model Ovi column densities in a $200 \mathrm{pc}$ radius sphere centered at the Sun at times 0.5 through $1.0 \mathrm{Myr}$ after the last SN event in the LB, overlaid by FUSE data from Savage \& Lehner (2006; SL2006), Welsh \& Lallement (2008; WL2008) and Barstow et al. (2010; BB2010) for observations agains white dwarfs in the up to $200 \mathrm{pc}$ from the Sun. The horizontal axis indicates the integration distance for the model and the star distance from the observational data.

$100 \mathrm{pc}$ in the first $0.5-0.6 \mathrm{Myr}$ since the last SN and growing to a maximum of $\log N(\mathrm{OvI})=13.3$ (in units of $\mathrm{cm}^{-2}$ ) in the next 0.2 Myr. For distances $>140 \mathrm{pc}$, the lines of sight are sampling gas not only from Loop I but also from the surrounding interstellar medium, hence the increase in the column density. It is clear that the modelled column densities fall within and follow the observed (with FUSE) data for path lengths greater than $50 \mathrm{pc}$. With increasing time, there is a slight increase in the amount of Ovi at smaller distances $(d<50 \mathrm{pc}$; orange and dark green lines at times 1.5 Myr and 2.0 Myr after the last SN event in the cavity).

For the first million years of evolution of the simulated bubbles, the majority of the LOS $(>75 \%)$ samples gas with $\log (N(\mathrm{OvI}))<13$, with this number reaching $99 \%$ and $95 \%$ levels at $0.5 \mathrm{Myr}$ and $0.7 \mathrm{Myr}$ after the last explosion (top panel of Fig. 3) and decreasing to some $80 \%$ in the next $0.3 \mathrm{Myr}$ (bottom panel of Fig. 3).

\subsection{Distribution of Li-like ion ratios}

Figure 4 displays the histograms of the LOS ratios of $\log [N(\mathrm{CIv}) / N(\mathrm{OvI})]$ and $\log [N(\mathrm{Nv}) / N(\mathrm{OvI})]$ in the LB between $0.5 \mathrm{Myr}$ and $1.0 \mathrm{Myr}$ after the last SN event. The fraction of LOS sampling gas with column density ratio $\log [N(\mathrm{Crv}) / N(\mathrm{OvI})]<$ -0.9 increases with time towards $87 \%$ at $0.6 \mathrm{Myr}$ and $93 \%$ at $0.7 \mathrm{Myr}$ (top panel, left column of Fig. 4) then decreases to less than $80 \%$ in the next $0.1 \mathrm{Myr}$ of the LB evolution (bottom panel, left column of the figure). The small fraction of LOS with $\log [N(\mathrm{Crv}) / N(\mathrm{OvI})]>-0.9$ is consistent with the detection by Welsh et al. (2010) of interstellar CIV in the LB with $N($ CIv $) \simeq 10^{12} \mathrm{~cm}^{-2}$ towards a star located at $74 \mathrm{pc}$ from the Sun. For $\log [N(\mathrm{Nv}) / N(\mathrm{OvI})]<-1.0$, the fraction of LOS sampling gas up to this threshold also increases to $89 \%$ and $92 \%$ levels at 0.6 and $0.7 \mathrm{Myr}$, respectively, after the last explosion in the LB, followed by a steep decrease to less than $70 \%$ in the next few hundred thousand years (right column of Fig. 4). This behaviour of the column density ratios in the simulated LB con-

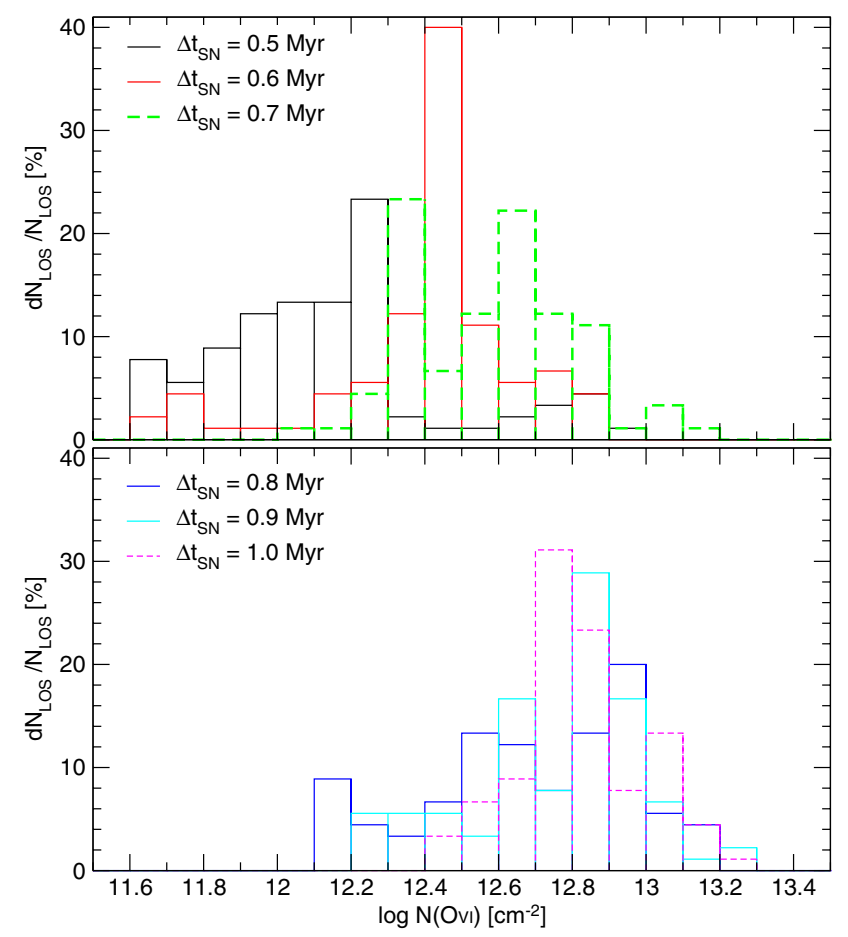

Fig. 3. Histograms of the $N(\mathrm{OvI})$ model predictions for LOS sampling only gas inside the cavity, i.e., LOS lengths $<100 \mathrm{pc}$, between 0.5 and 1.0 Myr after the last explosion in the LB cavity.

firms the presence of a very small amount of $\mathrm{CIV}$ and $\mathrm{Nv}$ ions and their uncorrelated distribution with OvI.

\section{Discussion and conclusions}

The work described here focuses on the detailed numerical evolution of a multi-supernova origin of the LB, following the studies by BA06 and AB09. A major difference from previous studies by other authors is that we (i) follow in a Eulerian fashion the joint evolution of the Local and Loop I bubbles in the turbulent ISM; (ii) use a three-dimensional high resolution supernova-driven ISM model to simulate a realistic ambient medium; (iii) calculate the time-dependent evolution of the complete ionization structure, and therefore also the corresponding time-dependent cooling function at each cell of the computational grid; and (iv) include the latest revision of solar abundances.

Three conditions determine the present evolution time $\left(t_{\mathrm{evol}}\right)$ of the LB: the $N(\mathrm{OvI})$ threshold of $8 \times 10^{12} \mathrm{~cm}^{-2}$ and the ratios $\log [n(\mathrm{CIv}) / n(\mathrm{OvI})<-0.9$ and $\log [N(\mathrm{Nv}) / N(\mathrm{OvI})]<-1.0$. A comparison to be between the LOS measurements at different times in the simulated LB indicates that $t_{\mathrm{evol}}$ can be constrained to be between $0.5 \mathrm{Myr}$ and $0.8 \mathrm{Myr}$ since the last $\mathrm{SN}$ explosion. The other times are excluded because they do not satisfy simultaneously the three threshold conditions, therefore, contradicting Copernicus, IUE, and GHRS-HST observations. It should be kept in mind, however, that the age of the LB, solely based on the simulated Ovi, is between 0.5 Myr and 1.1 Myr since the last $\mathrm{SN}$ event. The derived LB present age corresponds to a delay of at most 0.3 Myr (0.6 Myr if we only consider the Ovi distribution) compared to the $0.5 \mathrm{Myr}$ obtained in the CIE simulations of BA06 and AB09.

The comparison of Li-like ions between observations and NEI simulations pins down for the first time the age of the LB reliably well, which was impossible in previous CIE simulations. 

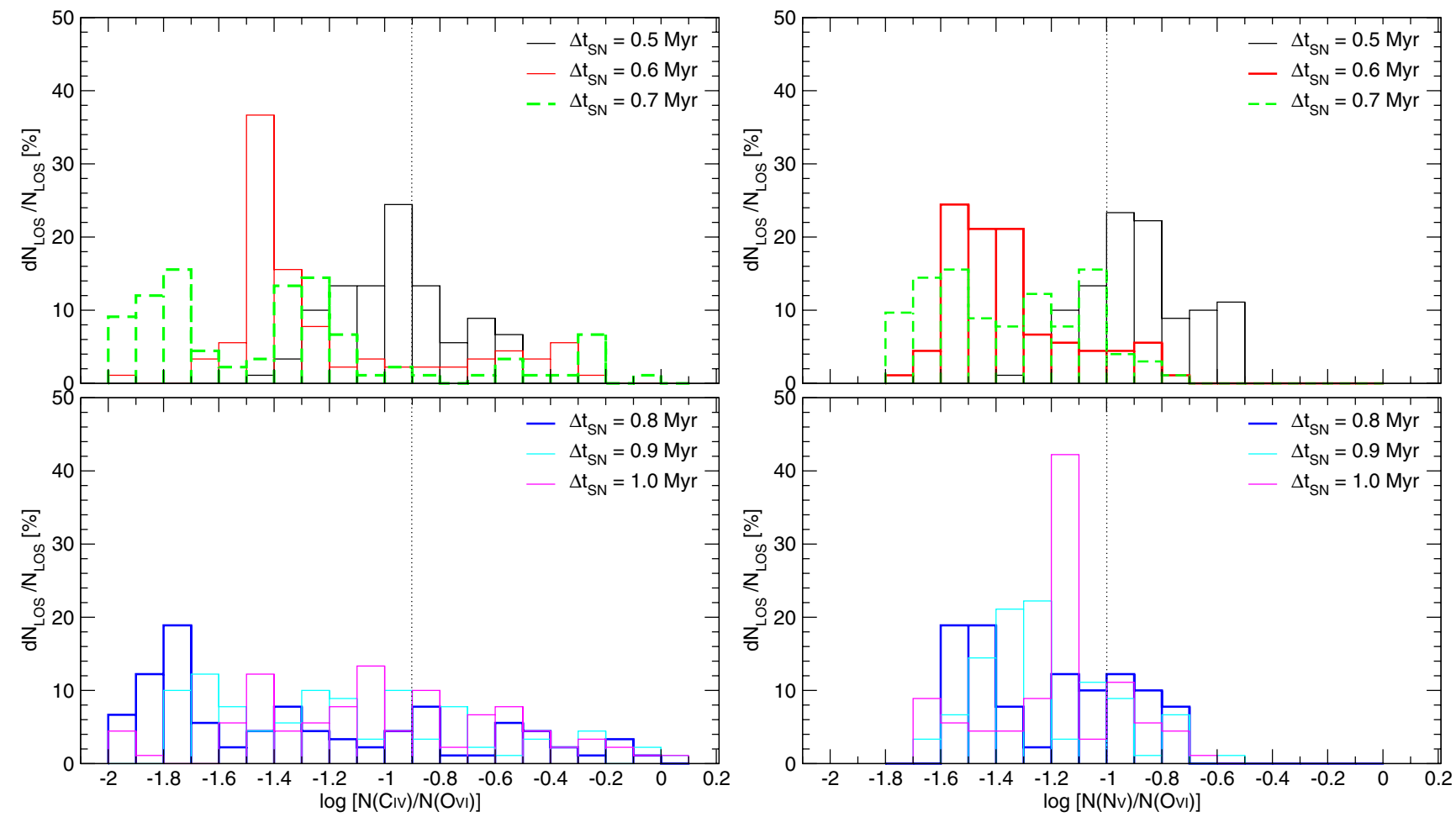

Fig. 4. Histogram of the LOS measurements of the column density ratios $N(\mathrm{CIV}) / N(\mathrm{OvI})$ (left panel) and $N(\mathrm{Nv}) / N(\mathrm{OvI})($ right panel) inside the simulated LB $\left(l_{\mathrm{LOS}}<100 \mathrm{pc}\right)$ for $0.5-1.0 \mathrm{Myr}$ after the last SN explosion. The vertical dotted line marks the critical column density ratios of -0.9 (left) and -1.0 (right), respectively (for details see text).

In particular, modelling the densities of different ions with different ionization and recombination timescales gives abundanceindependent results. Moreover, since spectral resolution in our models is not an issue, we will be able in the near future to also compare theoretical line widths to observations.

The small column densities observed over a wide range of temperatures in the simulations are the result of delayed recombination, which also manifests itself in X-ray emission at low temperatures, as we will discuss in the next few papers of the series. Finally, we note that we have performed calculations of the distribution of ${ }^{60} \mathrm{Fe}$ in the solar neighbourhood and compared the sequence of explosions with the measured iron peak in the deep sea ferromanganese crust (Knie et al. 2004), including the new half lifetime of Rugel et al. (2009), and found excellent agreement in the time sequences (Breitschwerdt et al., in prep.). Further developments of the model comprising the inclusion of magnetic field and cosmic rays will also be explored in papers to follow.

Acknowledgements. We would like to thank the referee, Don Cox, for his detailed reports, constructive criticism, and valuable suggestions that allowed us to improve this letter significantly. The simulations were carried out at the Milipeia Supercomputer (Center for Computational Physics, University of Coimbra) and at the ISM-Cluster of the Computational Astrophysics Group (Dept. of Mathematics, University of Évora). This research is supported by the FCT (Portugal) project PTDC/CTE-AST/70877/2006.

\section{References}

Asplund, M., Grevesse, N., \& Sauval, A. J., \& Scott, P. 2009, ARA\&A, 47, 481 Barstow, M. A., Boyce, D. D., Welsh, B. Y., et al. 2010, ApJ, 723, 1762
Breitschwerdt, D., \& de Avillez, M. A. 2006, A\&A, 452, L1 (BA06)

Berghöfer, T., \& Breitschwerdt, D. 2002, A\&A, 390, 299

Bertin, P., Vidal-Madjar, A., Lallement, R., Ferlet, R., \& Lemoine, M. 1995, A\&A, 302, 889

Bruhweiler, F. C., Kondo, Y., \& McCluskey, G. E. 1980, ApJ, 237, 19

Cox, D. P., \& Anderson, P. R. 1982, ApJ, 253, 268

de Avillez, M. A., \& Breitschwerdt, D. 2004, A\&A, 425, 899

de Avillez, M. A., \& Breitschwerdt, D. 2009, ApJ, 697, L158 (AB09)

de Avillez, M. A., Spitoni, E., \& Breitschwerdt, D. 2012, in Advances in Computational Astrophysics: Methods, Tools and Outcomes, ed. R. Dolcetta, M. Limongi, A. Tornambe, ASP Conf. Ser., in press

Egger, R. 1998, In IAU Colloq. 166, Lect. Not. Phys., 506, 287

Freyberg, M., \& Breitschwerdt, D. 2003, AN, 324, 162

Fuchs, B., Breitschwerdt, D., de Avillez, M. A., Dettbarn, C., \& Flynn, C. 2006, MNRAS, 373, 993

Henley, D. B., Shelton, R. L., \& Kuntz, K. D. 2007, ApJ, 661, 304

Huang, J.-S., Songaila, A., Cowie, L. L., \& Jenkins, E. B. 1995, ApJ, 450, 163

Hurwitz, M., Sasseen, T. P., \& Sirk, M. M. 2005, ApJ, 623, 911

Innes, D. E., \& Hartquist, T. W. 1984, MNRAS, 209, 7

Jelinsky, P., Vallerga, J. V., \& Edelstein, J. 1995, ApJ, 441, 653

Jenkins, E. B. 1978, ApJ, 219, 845

Kafatos, M. 1973, ApJ, 182, 433

Knie, K., Korschinek, G., Faestermann, T., et al. 2004, Phys. Rev. Lett., 93, 171103

Maíz-Apellániz, J. 2001, ApJ, 560, L83

McCammon, D., Almy, R., Apodaca, E., et al. 2002, ApJ, 576, 188

Rugel, G. Faestermann, T., Knie, K., et al. 2009, Phys. Rev. Lett., 103, 072502

Sanders, W. T., Edgar, R. J., Kraushaar, W. L., McCammon, D., \& Morgenthaler, J. P. 2001, ApJ, 554, 694

Savage, B. D., \& Lehner, N. 2006, ApJS, 162, 134

Schmutzler, T., \& Tscharnuter, W. M. 1993, A\&A, 273, 318

Shelton, R., \& Cox, D. P. 1994, ApJ, 434, 599

Smith, R. K., \& Cox, D. P. 2001, ApJS, 134, 283

Welsh, B. Y., \& Lallement, R. 2008, A\&A, 490, 707

Welsh, B. Y., Wheatley, J., Siegmund, O. H. W., \& Lallement, R. 2010, ApJ, 712, L199

York, D. G. 1977, ApJ, 213, 43 